\title{
Cec and You Shall Find: Cecal Perforation in a Patient with COVID-19
}

\author{
loana Baiu ${ }^{1}$ Erna Forgó ${ }^{2} \cdot$ Cindy Kin $^{1} \cdot$ Thomas G. Weiser $^{1}$
}

Accepted: 23 December 2020 / Published online: 25 January 2021

(C) The Author(s), under exclusive licence to Springer Science+Business Media, LLC part of Springer Nature 2021

\section{Case Presentation and Evolution}

A 66-year-old man with morbid obesity (BMI 36), diabetes mellitus (HbA1c 9.4), hypertension, stage III chronic kidney disease (baseline creatinine 1.4), and obstructive sleep apnea (on home CPAP) was transferred to our emergency room due to pneumoperitoneum. Two weeks prior to transfer, he was evaluated at a community hospital emergency room for complaints of dyspnea, nausea, vomiting, abdominal pain, and diarrhea. A CT scan of abdomen and pelvis revealed diverticulosis without evidence of diverticulitis; the visualized cuts of the lower chest revealed diffuse lung infiltrates. He was diagnosed with COVID-19 by PCR testing and started on a 5-day course of dexamethasone; his abdominal pain was attributed to his viral infection. One week later, he had worsening diarrhea and abdominal pain and tested positive for Clostridioides difficile, for which oral vancomycin treatment was started. Six days into this antibiotic course, he developed sudden onset severe abdominal pain; a CT now showed extensive pneumoperitoneum without a clear source. His vital signs were within normal limits, and he was started on broad-spectrum antibiotics and transferred to Stanford Hospital for surgical management. On arrival, he had a pulse of 91 , blood pressure of $161 / 86$, respiratory rate of 17 , oxygen saturation of $95 \%$ on room air, and temperature of $36.9^{\circ} \mathrm{C}$.

Thomas G. Weiser

tweiser@stanford.edu

Ioana Baiu

ibaiu@stanford.edu

Erna Forgó

eforgo@stanford.edu

Cindy Kin

cindykin@stanford.edu

1 Department of Surgery, Stanford University School of Medicine, 300 Pasteur Drive, H3563, Stanford, CA 94305, USA

2 Department of Pathology, Stanford University School of Medicine, 300 Pasteur Drive, L235, Stanford, CA 94305, USA
On examination, he was obese and dyspneic while sitting upright; his abdomen was very distended but soft, with mild diffuse tenderness throughout; he had no evidence of peritonitis. His laboratory tests revealed a WBC $10 \mathrm{k} / \mu \mathrm{L}$, platelets $408 \mathrm{k} / \mu \mathrm{L}$, creatinine $1.4 \mathrm{mg} / \mathrm{dL}$, and whole blood lactate $1.5 \mathrm{mmol} / \mathrm{L}$. His outside hospital CT scan (Fig. 1) showed massive pneumoperitoneum concentrated mostly in the epigastrium. The differential diagnosis at this time included perforated peptic ulcer versus colonic perforation based on the extent of the free air. We proceeded with an exploratory laparotomy (Fig. 2).

The patient was intubated using our COVID-19 hospital protocol which included intubation in a negative pressure isolation room and then transported to the operating room under mechanical ventilation with a viral filter circuit attached to the endotracheal tube. A $5 \mathrm{~mm}$ epigastric incision was made after which the pneumoperitoneum was evacuated using a closed suction catheter in order to minimize aerosolization. An upper midline incision was then used to enter the abdomen, and the stomach, duodenum, and lesser sac were examined carefully. There was no evidence of perforation or inflammation. The laparotomy was extended below the umbilicus revealing murky fluid and inflammatory changes in the right lower quadrant. The right colon and transverse colon were dilated and dusky. Upon closer inspection, a small perforation was noted in the distal cecum with slow active spillage. Given his history of $C$. difficile, a subtotal colectomy that involved the right and transverse colon as well as the splenic flexure and proximal descending colon was performed. A long Hartmann pouch was created and brought to the skin as a mucous fistula; similarly, an end ileostomy was created and matured. The mucosa of the colon in the mucous fistula appeared normal and healthy.

Pathological review of the colon demonstrated diffuse serositis and transmural perforation of the cecum without other evidence indicative of active infection. Gross pathology examination of the entire specimen demonstrated bowel with a focal area of hemorrhage and exudate on the serosal surface, with an isolated perforation. The ileal and colonic segments revealed areas of denuded mucosa with 


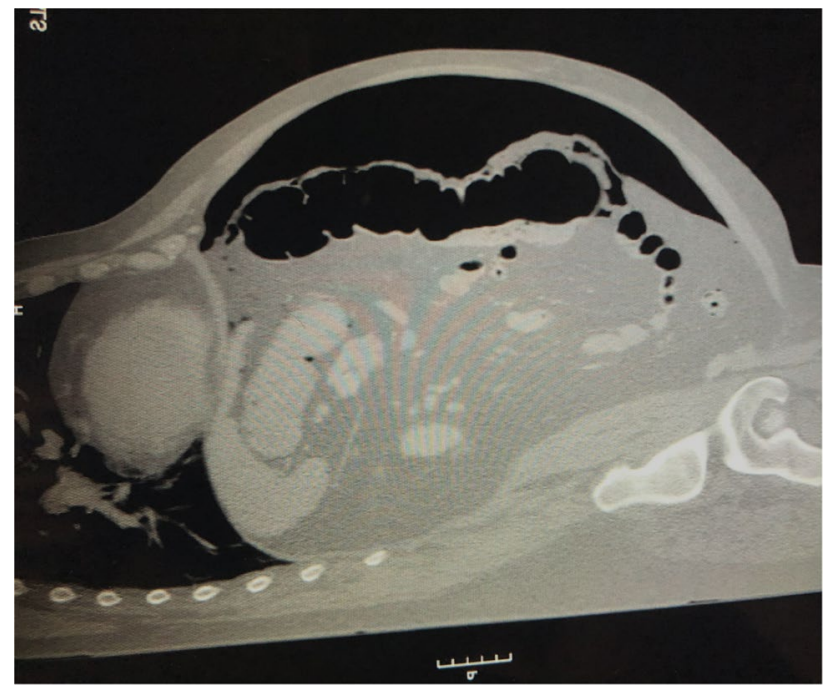

Fig. 1 CT scan in sagittal section showing pneumoperitoneum

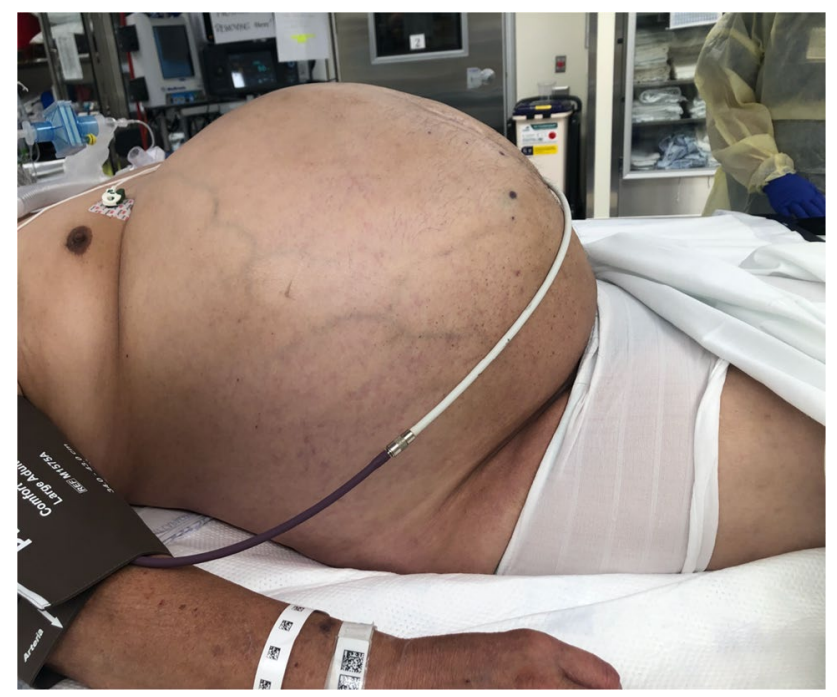

Fig. 2 Preoperative exam with marked abdominal distension correlating with pneumoperitoneum seen on CT

patchy exudative adhesions and hemorrhage close to the perforation. Microscopic examination of the terminal ileum (Fig. 3a), right colon and proximal transverse (Fig. 3b), and splenic flexure (Fig. 3c) resections demonstrated serositis and serosal adhesion (Fig. 3d) without transmural inflammation or mucosal disruption. The colon was otherwise without histologic features to suggest a specific etiology for the perforation, such as ischemia, mucosal injury, inflammation, or any manifestation of $C$. difficile infection, nor was there evidence of microthrombosis as a cause.

These findings suggested that the colonic perforation was spontaneous and not related to the $C$. difficile infection for which he had already undergone treatment. The patient's postoperative recovery was delayed by an ileus that eventually spontaneously resolved, as well as bilateral deep venous thrombosis and pulmonary emboli despite appropriate venous thromboprophylaxis therapy with enoxaparin. A vascular ultrasound for suspected DVT revealed rouleaux formation, which was thought consistent with the hypercoagulability described in patients with COVID-19.

\section{Discussion}

In the setting of a history of $C$. difficile, a total abdominal colectomy is indicated for patients for whom there is concern for toxic megacolon [1]. Typically, a Hartmann segment is created and either left in place in the pelvis or brought up to the skin as a mucous fistula. The reasons for creating a mucous fistula are threefold: (1) An intraabdominal staple line leak is avoided by having the mucous fistula already exteriorized; (2) the rate of feasible and safe re-anastomosis is increased as it is significantly easier to perform an ileocolic anastomosis to a long Hartmann rather than to a short rectal stump; and (3) it facilitates both antegrade and retrograde vancomycin enemata in the setting of active infection. This patient, however did not have any signs or symptoms of toxic megacolon, such as hemodynamic instability, hypotension, tachycardia, or inotropic requirement. Following creation of the mucous fistula, the colonic mucosa was visualized and noted to be entirely normal, not consistent with $C$. difficile colitis. Overall, the presentation was most consistent with colonic small vessel thrombosis with resultant ischemia in the setting of active COVID-19.

Our knowledge of COVID-19 and its full pathophysiologic effects on the gastrointestinal tract is still being elucidated. It is well known that coronaviruses bind to exoenzymes such as dipeptidyl peptidase 4 (DPP4) or angiotensin-converting enzyme (ACE) 2, typically located in the pulmonary and the gastrointestinal epithelia with subsequent invasion and tissue destruction, which explains why many infected patients develop respiratory symptoms and/or abdominal pain and diarrhea, and why viral RNA is detected in stool samples of infected patients [2]. Tropism for the gastrointestinal tract can be explained by the presence of the abundance of ACE2 in the lumen, the principal receptors for SARS-CoV-2 [3]. In fact, ACE2 expression is 100-fold higher in the gastrointestinal tract than in the lung, and it is also present in the vascular endothelium. In a study of 1070 specimens from 205 infected patients, SARSCoV-2 RNA was detected in $93 \%$ of bronchoalveolar lavage specimens, $72 \%$ sputum samples, $63 \%$ of nasal swabs, $46 \%$ of bronchoscopic brush biopsies, $32 \%$ of pharyngeal swabs, $29 \%$ of fecal specimens, $1 \%$ of blood samples, and in $0 \%$ of urine samples [4]. A large international study of 25,210 COVID-19 patients noted that $18.6 \%$ have gastrointestinal 

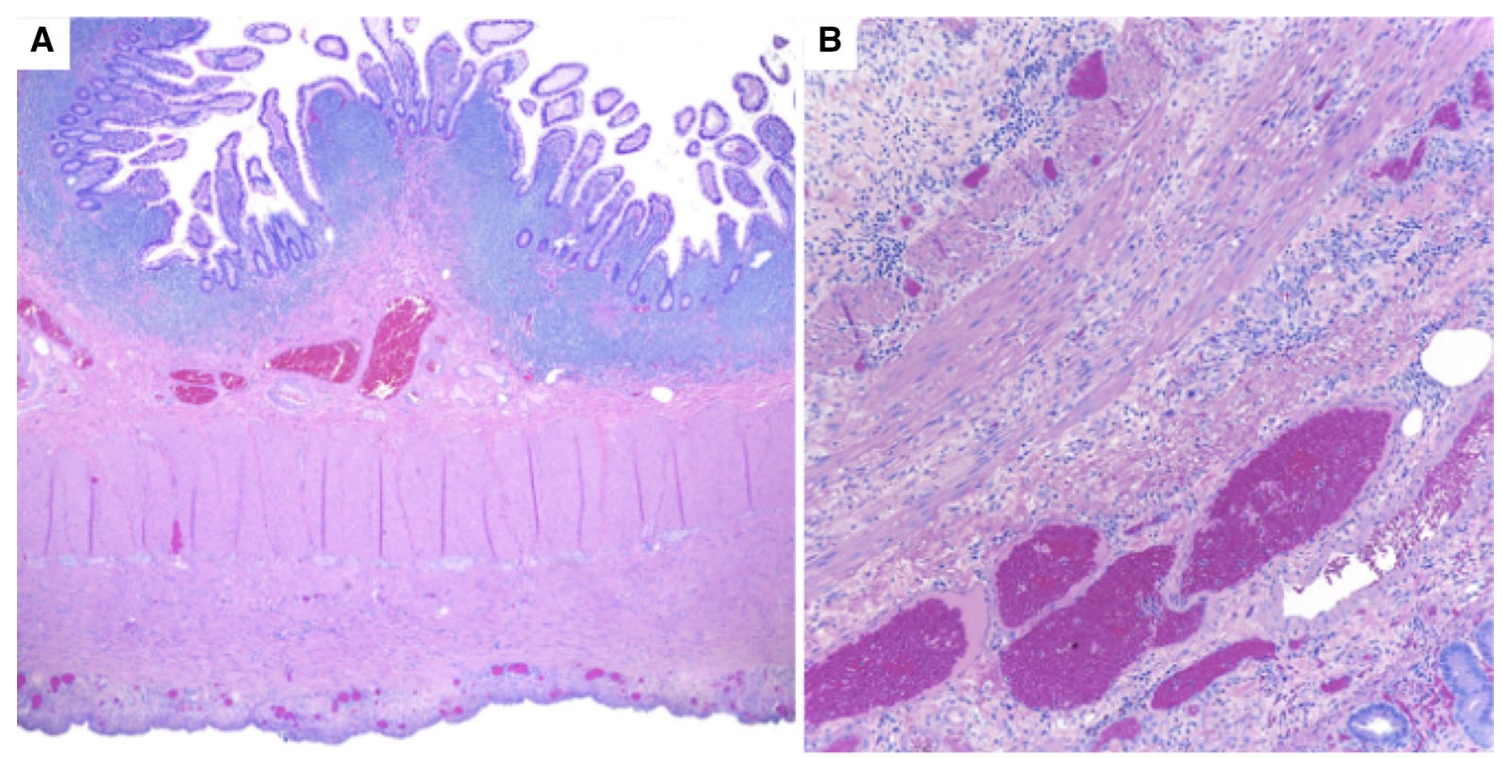

C

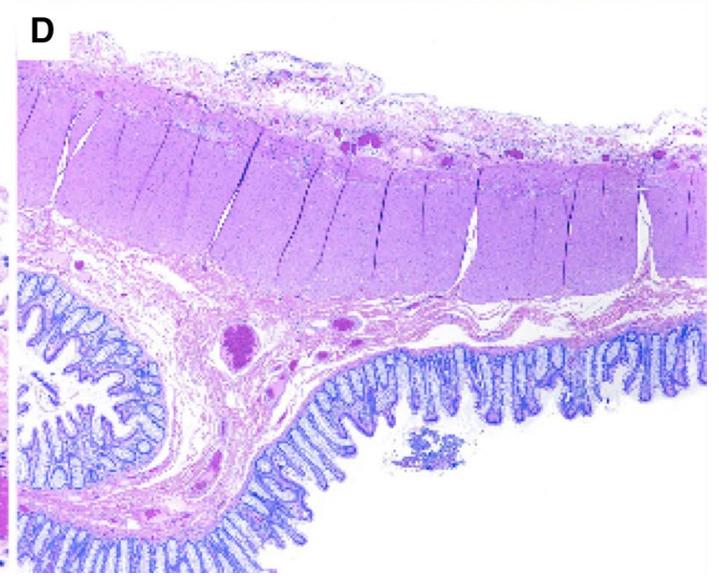

Fig. 3 Microscopic examination of the terminal ileum (a), right colon and proximal transverse (b), splenic flexure (c) resections demonstrated serositis and serosal adhesion (d) without transmural inflammation or mucosal disruption

symptoms (anorexia 26\%, diarrhea $13.5 \%$, nausea/vomiting $9.4 \%$, abdominal pain 5.7\%) and that the presence of any of the above correlates with disease severity and ICU admission [5]. Furthermore, SARS-CoV-2 infection has been associated with a state of hypercoagulability presumed to be caused by endothelial injury, immobilization, and activation of prothrombotic factors [6, 7]. When 92 critically ill patients with acute respiratory distress syndrome (ARDS) were compared with 92 propensity matched ARDS patients with COVID-19, the latter group had significantly more abnormal aminotransferases ( $55 \%$ vs $27 \%, p<0.001$ ), severe ileus ( $48 \%$ vs $22 \%, p<0.001$ ), and bowel ischemia ( $4 \%$ vs $0 \%, p=0.04)$ [8]. Three of the patients who had bowel ischemia required bowel resection; pathology demonstrated fibrin thrombi in the affected vasculature. A meta-analysis of 6,686 patients with COVID-19 confirmed that the pooled prevalence of digestive symptoms is $15 \%$ with $19 \%$ of patients having abnormal liver function [9]. Viral shedding via the fecal route has been reported in multiple studies to contribute to the transmission of the viral particles [10].

Interestingly, there are radiographic signs of infection. In a retrospective study of 412 patients (average age 57 years) with COVID-19 (58.5\% men, $41.5 \%$ women), $17 \%$ had cross-sectional imaging of which $30 \%$ had anatomic abnormalities of the GI tract, such as thickening, bowel wall ischemia, pneumatosis, or portal venous gas in the absence of another diagnosis [11]. More recently, a systematic review noted that $20 \%$ of patients with COVID-19 have radiologic evidence of infection, such as large bowel wall thickening, fluid filled colon, pneumatosis intestinalis, pneumoperitoneum, intussusception, and ascites [12].

To date, there have been three case reports of patients with gastrointestinal perforations in the setting of COVID19 infection, which highlights the possible etiology of acute abdominal distention and presence of free air with 
complicating perforation in the absence of other infectious or mechanical causes [13-15].

In summary, this patient with active COVID-19 infection suffered a spontaneous cecal perforation without evidence for infectious colitis, as well as the hypercoagulable state evidenced by pulmonary embolism, and ultrasound evidence of rouleaux formation with clinical evidence of thromboembolism.

\section{Compliance with Ethical Standards}

Conflict of interest The authors have no extramural funding or conflicts of interest relevant to this work.

\section{References}

1. Berman L, Carling T, Fitzgerald TN, Bell RL, Duffy AJ, Longo WE et al. Defining surgical therapy for pseudomembranous colitis with toxic megacolon. J ClinGastroenterol 2008;42:476-480

2. Tian Y, Rong L, Nian W, He Y. Review article: gastrointestinal features in COVID-19 and the possibility of faecal transmission. Aliment PharmacolTher 2020;51:843-851

3. Kotfis K, Skonieczna-Żydecka K. COVID-19: gastrointestinal symptoms and potential sources of SARS-CoV-2 transmission. AnaesthesiolIntensTher 2020;52:171-172

4. Wang W, Xu Y, Gao R, Lu R, Han K, Wu G et al. Detection of SARS-CoV-2 in different types of clinical specimens. JAMA 2020;328:1843-1844

5. Zhao Y, Cao Y, Wang S, Cai K, Xu K. COVID-19 and gastrointestinal symptoms. Br J Surg 2020;107:e382-e383

6. Abou-Ismail MY, Diamond A, Kapoor S, Arafah Y, Nayak L. The hypercoagulable state in COVID-19: incidence, pathophysiology, and management. Thrombosis Res 2020;194:101-115
7. Singhania N, Bansal S, Nimmatoori DP, Ejaz AA, McCullough PA, Singhania G. Current overview on hypercoagulability in COVID-19. Am J Cardiovasc Drugs 2020;20:393-403

8. El Moheb M, Naar L, Christensen MA, Kapoen C, Maurer LR, Farhat $\mathrm{M}$ et al. Gastrointestinal complications in critically ill patients with and without COVID-19. JAMA 2020;324:1899-1901

9. Mao R, Qiu Y, He J-S, Tan J-Y, Li X-H, Liang J et al. Manifestations and prognosis of gastrointestinal and liver involvement in patients with COVID-19: a systematic review and meta-analysis. Lancet GastroenterolHepatol 2020;5:667-678

10 Parasa S, Desai M, ThoguluvaChandrasekar V, Patel HK, Kennedy KF, Roesch T et al. Prevalence of gastrointestinal symptoms and fecal viral shedding in patients with coronavirus disease 2019: a systematic review and meta-analysis. JAMA Netw Open 2020;3:e2011335

11. Bhayana R, Som A, Li MD, Carey DE, Anderson MA, Blake $\mathrm{MA}$ et al. Abdominal imaging findings in COVID-19: preliminary observations. Radiology 2020;297:E207-E215

12 Lui K, Wilson MP, Low G. Abdominal imaging findings in patients with SARS-CoV-2 infection: a scoping review. AbdomRadiol. 2020. https://doi.org/10.1007/s00261-020-02739-5.

13. Rojo M, Cano-Valderrama O, Picazo S, Saez C, Gómez L, Sánchez C et al. Gastrointestinal perforation after treatment with tocilizumab: an unexpected consequence of COVID-19 pandemic. Am Surg 2020;86:565-566

14. De Nardi P, Parolini DC, Ripa M, Racca S, Rosati R. Bowel perforation in a Covid-19 patient: case report. Int J Colorectal Dis 2020;35:1797-1800

15. CorrêaNeto IJF, Viana KF, da Silva MBS, da Silva LM, de Oliveira G, Cecchini AR da $S$ et al. Perforated acute abdomen in a patient with COVID-19: an atypical manifestation of the disease. J Coloproctol 2020;40:269-272

Publisher's Note Springer Nature remains neutral with regard to jurisdictional claims in published maps and institutional affiliations. 